\title{
Numerical Studies of Biocontrol Efficacies of Foliar Plant Pathogens in Relation to the Characteristics of a Biocontrol Agent
}

\author{
X.-M. Xu, N. Salama, P. Jeffries, and M. J. Jeger \\ First author: Plant Pathology, East Malling Research, New Road, East Malling, ME19 6BJ, UK; second and fourth author: Division of \\ Biology, Imperial College London, Silwood Park Campus, Ascot, SL5 7PY, UK; and third author: School of Biosciences, University of \\ Kent, Canterbury CT2 7NJ, UK. \\ Accepted for publication 26 April 2010.
}

\begin{abstract}
Xu, X.-M., Salama, N., Jeffries, P., and Jeger, M. J. 2010. Numerical studies of biocontrol efficacies of foliar plant pathogens in relation to the characteristics of a biocontrol agent. Phytopathology 100:814-821.

A previously published generic mathematic model has been used in a numerical study to understand the dynamics of foliar pathogens in relation to mechanisms, and timing and coverage of biocontrol agent (BCA) applications. With the model parameter values used, it was demonstrated that a BCA possessing either competition or induced resistance as the main mechanism of biological control was more effective in reducing disease development than a BCA with either mycoparasitism or antibiosis as its mechanism. Application coverage, ranging from 50 to $90 \%$, had

biocontrol efficacy for those with competition or induced resistance as their main mechanism than those with mycoparasitism and antibiosis. Biocontrol efficacy was greatest for a single BCA combining competition with mycoparasitism or antibiosis. The efficacy for a single BCA combining induced resistance with competition critically depended on application time; the efficacy was greatly reduced for delayed applications. The present study suggests that development of an effective strategy for BCA application is critically dependent upon our quantitative understanding of several key biocontrol processes and their interactions. Without reliable quantitative estimation of these processes, it is impossible to make quantitative predictions about biological control and hence to optimize BCA application strategies.
\end{abstract} little effect on biocontrol efficacy, particularly for a BCA with competition and induced resistance as the main mechanism of biocontrol. Conversely, delayed application of BCA had more profound effects on

Sustainable biological control of plant pathogens depends on efficient exploitation of naturally occurring microorganisms. The complex nature of phyllosphere ecology has, so far, militated against the development of successful biocontrol agents (BCAs) for foliar plant pathogens. However, there is optimism concerning future prospects $(8,21)$ and some demonstrated successes in greenhouse cultivation $(9,17)$. The key to investigating dynamics of a biocontrol system is to understand the mechanisms involved in biocontrol, how they interact, and how they are manifested at different hierarchical levels. The principal mechanisms involved include mycoparasitism, antibiosis, competition and induced resistance $(1,5,18)$; additional mechanisms are through hypovirulence mediated through fungal viruses (20) and inhibition of enzymes involved in plant pathogenicity (14).

Mathematical modeling can be used to study disease dynamics in relation to BCA dynamics through the interactions defined by the biocontrol mechanisms. Several approaches have been used to model plant-pathogen-BCA systems, ranging from simple infection-BCA dose relationships (3) to relative complex simulation models $(15,16)$. We have recently developed a generic modeling framework to understand the dynamics of a biocontrol system in which BCA and foliar pathogen dynamics were linked together through the principle biocontrol mechanisms of competition, antibiosis, mycoparasitism and induced resistance (13). Qualitative analysis of the model showed that the rates of a BCA colonizing

Corresponding author: X.-M. Xu: E-mail address: xiangming.xu@emr.ac.uk

*The $e$-Xtra logo stands for "electronic extra" and indicates that Figures 1 to 7 appear in color online.

doi:10.1094/PHYTO-100-8-0814

(c) 2010 The American Phytopathological Society
Additional keywords: antibiosis, biocontrol mechanism, competition, induced resistance, mycoparasitism. diseased and/or healthy plant tissues and the time that the BCA remains active are two of the more important factors in determining the final outcome of a biocontrol system.

In some cases, individual biocontrol mechanisms have been shown to be predominant, but there are also many instances where more than one mechanism may operate in a given BCA isolate such as Trichoderma harzianum T39, e.g., mycoparasitism, antibiosis, competition, and induced resistance $(4,23)$, or where mixed BCA populations combine a range of mechanisms $(6,10,11)$. The relative significance of each mechanism may vary with circumstances but it is clear that multiple mechanisms are involved in biocontrol systems in field conditions. There have been few systematic studies of comparing biocontrol efficacies when more than one biocontrol mechanism is involved. A study using detached strawberry leaves demonstrated that biocontrol of Botrytis cinerea was improved and that variability in the efficacy was reduced when two BCAs (one yeast and one bacterium) combining several biocontrol mechanisms were applied together (11).

In this paper, we have modified a published model (13) and then carried out numerical evaluation of the model to study the dynamics of biological control of foliar pathogens. Specifically, we aimed to investigate the effects of the following factors on disease development: (i) individual biocontrol mechanisms, (ii) coverage of BCA applications, (iii) timing of BCA application, and (iv) multiple biocontrol mechanisms. These aspects are critically important for developing and implementing strategies of using BCAs in commercial agriculture.

\section{THEORY AND APPROACHES}

Model description. The present model for numerical evaluation of biocontrol of foliar pathogens was based on the generic 
model developed by Jeger et al. (13). In this generic model, a standard susceptible-infected-removed (SIR) model for hostpathogen dynamics is coupled with one for the pathogen-BCA dynamics; this deterministic model does not consider spatial heterogeneity. Host tissue is divided into the following mutually exclusive categories: healthy susceptible $\left(H_{s}\right)$, healthy immune to the pathogen $\left(H_{r}\right)$, infectious disease $(I)$, removed disease $(R)$, and area occupied by a BCA $\left(H_{b}\right)$. These state variables were defined in the linked differential equations:

$\frac{d H_{s}}{d t}=-b I H_{s}-\left(c_{1}+d\right) H_{b} H_{s}+\frac{H_{s}+H_{r}}{1-H_{b}} f H_{b}$

$\frac{d H_{r}}{d t}=H_{b}\left(d H_{s}-c_{1} H_{r}\right)$

$\frac{d H_{b}}{d t}=H_{b}\left[c_{1}\left(H_{s}+H_{r}\right)+c_{2}\left(1-H_{s}-H_{r}-H_{b}\right)-f\right]$

$\frac{d I}{d t}=I\left(b H_{s}-c_{2} H_{b}-h\right)$

with the equation for $R=1-\left(H_{s}+H_{r}+H_{b}+I\right)$. Redundant. The definition and dimension of state variables and parameters are given in Table 1.

We have modified this generic model in two aspects for the present numerical study. First, we have introduced a new parameter $(e)$, the rate of immune host tissue reverting directly to healthy, susceptible tissue. This parameter was not included in the original model (13) for the mathematical tractability to obtain an analytical solution of the steady state (but this parameter does not affect the qualitative characteristics of biocontrol systems-Figure 9 in literature citation 13). Next, in the original model, a proportion of $H_{b}$ was reverted to removed disease $(R)$ with a proportion to healthy, susceptible tissue $\left(H_{s}\right)$. This is appropriate when both the $\mathrm{BCA}$ and pathogen are introduced into the system in very small quantities. However, it is inappropriate when we consider inundative application of BCA because, in this scenario, a proportion of host tissue instantaneously colonized by a BCA (due to inundative application) would move directly to $R$. Thus, in this modification we have moved this proportion to $H_{s}$ only (a certain proportion of the BCA treated area being reverted back to healthy, susceptible fails to establish). Thus the modified model is

$\frac{d H_{s}}{d t}=-b I H_{s}-\left(c_{1}+d\right) H_{b} H_{s}+e H_{r}+f H_{b}$

$\frac{d H_{r}}{d t}=H_{b}\left(d H_{s}-c_{1} H_{r}\right)-e H_{r}$

$\frac{d H_{b}}{d t}=H_{b}\left[c_{1}\left(H_{s}+H_{r}\right)+c_{2}\left(1-H_{s}-H_{r}-H_{b}\right)-f\right]$

$\frac{d I}{d t}=I\left(b H_{s}-c_{2} H_{b}-h\right)$
Numerical evaluation. We have conducted a numerical evaluation to investigate the effect of (i) individual biocontrol mechanisms, (ii) coverage of BCA application, (iii) timing of BCA application, and (iv) multiple biocontrol mechanisms. Table 1 gives sets of parameter values for a BCA with a specific biocontrol mechanism used in the numerical evaluations. Numerical simulation (by the Modified Extended Backward Differentiation Equation Implicit method) was carried out in Maple 11.

(i) Four biocontrol mechanisms were considered: antibiosis, competition, induced resistance, and mycoparasitism. Each mechanism is characterized by a combination of model parameters (Table 1). For example, a BCA with competition as its main mechanism is assumed to have a fast rate of colonizing healthy host tissue $\left(c_{1}\right)$ but with very little effect on colonizing diseased tissue or induced resistance.

(ii) Five levels $(p)$ of BCA coverage were considered: 50, 60, 70,80 , and $90 \%$. For a BCA in which the main biocontrol mechanism does not include antibiosis or mycoparasitism, only a proportion $(p)$ of healthy susceptible tissue was allocated to the BCA with no changes made to the infectious and removed disease categories. On the other hand, when a BCA whose main biocontrol mechanism includes antibiosis or mycoparasitism was applied, a proportion $(p)$ of all types of host tissues including infectious and removed disease was allocated to the BCA.

(iii) A BCA was applied 0, 14, and 28 days after introduction of the pathogen. In all cases, introduction of the pathogen gave an $I_{0}$ value of $0.1 \%$. For introduction of the BCA at day 0 , a proportion of the healthy host tissue (i.e., $p \times 99.9 \%$ ) was allocated to the BCA. With introduction at 14 or 28 days after the pathogen introduction, the proportion $(p)$ of host tissue allocated to the BCA depended on biocontrol mechanisms as described under (ii).

(iv) A BCA with two main biocontrol mechanisms was defined by combining the larger values of model parameters for each individual mechanism (Table 1). Thus, for example, a BCA with mycoparasitism and competition was defined to have the following set of parameter values: $c_{1}=0.4, c_{2}=0.4, f=0.2, d=0.01$, and $e=0.0$.

Three categories of pathogen aggressiveness (high, medium, and low) were used in the numerical evaluations as defined in Table 1. The model (equation 2) was evaluated for 200 days at a daily integration step.

\section{RESULTS}

Epidemic development of a pathogen with low aggressiveness was greatly suppressed by a BCA with any given set of BCA parameter values (Table 1), except for mycoparasitism. For a pathogen with medium aggressiveness the effect of a BCA on epidemic development was qualitatively the same as for a pathogen with high aggressiveness. Therefore, only results for a pathogen of high aggressiveness are presented.

TABLE 1. Parameter descriptions and their values used in numerical evaluation; each set of biocontrol agent (BCA) parameter values represents a BCA with a single specific biocontrol mechanism

\begin{tabular}{|c|c|c|c|c|c|}
\hline \multicolumn{2}{|c|}{$\mathrm{BCA}$ rate parameter $\left(\right.$ time $\left.^{-1}\right)$} & \multirow{2}{*}{$\frac{\text { Mycoparasitism }}{0.01}$} & \multirow{2}{*}{$\frac{\text { Competition }}{0.4}$} & \multirow{2}{*}{$\frac{\text { Antibiosis }}{0.2}$} & \multirow{2}{*}{$\frac{\text { Induced resistance }}{0.2}$} \\
\hline$c_{1}$ & Colonizing healthy tissue & & & & \\
\hline$c_{2}$ & Colonizing diseased tissue & 0.4 & 0.01 & 0.2 & 0.01 \\
\hline$f$ & Mortality (to susceptible) & 0.2 & 0.2 & 0.2 & 0.2 \\
\hline$e$ & From immune to susceptible & 0 & 0 & 0.1 & 0.1 \\
\hline
\end{tabular}

Pathogen aggressiveness

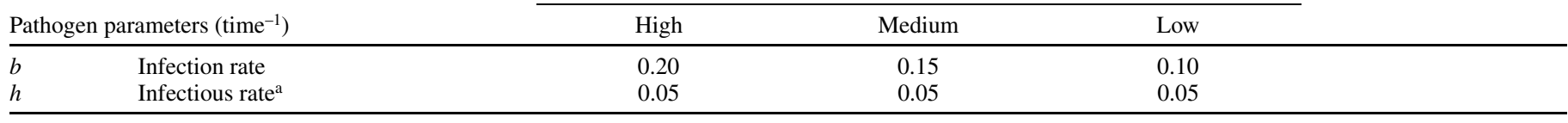

${ }^{\text {a }}$ Inverse of infectious period. Note that $b / h$ is $R_{0}$ in absence of a BCA, so higher aggressiveness can be achieved by increasing $b$ or decreasing $h$. 
Efficacy of a BCA with a single biocontrol mechanism at $50 \%$ coverage. When a BCA with a single biocontrol mechanism was applied at the same time as pathogen introduction, disease suppression was least and greatest over the first 100 days when mycoparasitism or competition was the main mechanism, respectively (Fig. 1). For example, it took 30 days for total disease to reach $10 \%$ without the BCA compared with 41 and 93 days with mycoparasitism and competition, respectively, as the main BCA mechanism (Fig. 1). As for the competition mechanism, to a lesser extent induced resistance had only delayed epidemic development. Both antibiosis and mycoparasitism had longer lasting effects, in the latter case with damped oscillations in disease dynamics. Biocontrol efficacy, calculated as the reduction in disease, expressed as percentage of disease in the absence of BCA, varied greatly with biocontrol mechanisms over time (Fig. 2).

As expected, biocontrol efficacy varied greatly with BCA mortality (Fig. 3). For $f=0.1$, a BCA successfully suppressed disease development, except in the case of mycoparasitism. However, the effect of $f$ in the case of mycoparasitism gradually became more pronounced (Fig. 3) and the disease development was constrained, albeit cyclically, even at higher BCA mortality rates.

Efficacy of a BCA with a single mechanism applied at varying coverage. The effect of varying coverage was generally small for a BCA with any single biocontrol mechanism (Fig. 1) when the BCA was applied at the same time as pathogen introduction. This was particularly so for competition and induced resistance. For example, with competition as the main biocontrol mechanism applying a BCA at $50 \%$ coverage led to a delay of 88 days for the total disease to reach $40 \%$; increasing the coverage to $90 \%$ led to an additional delay of 7 days only. For mycoparasitism, the initial effect of varying coverage was also small but gradually increased as the cycles in disease developed (Fig. 1).

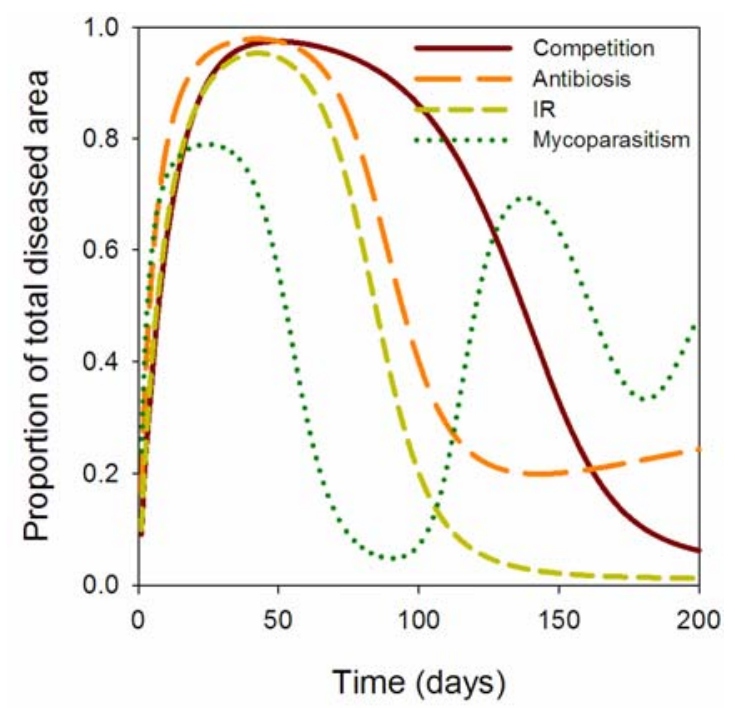

Fig. 2. Estimated biocontrol efficacy over time in relation to biocontrol mechanism when a biocontrol agent was applied at the same time as introduction of a pathogen of high aggressiveness at $50 \%$ coverage.
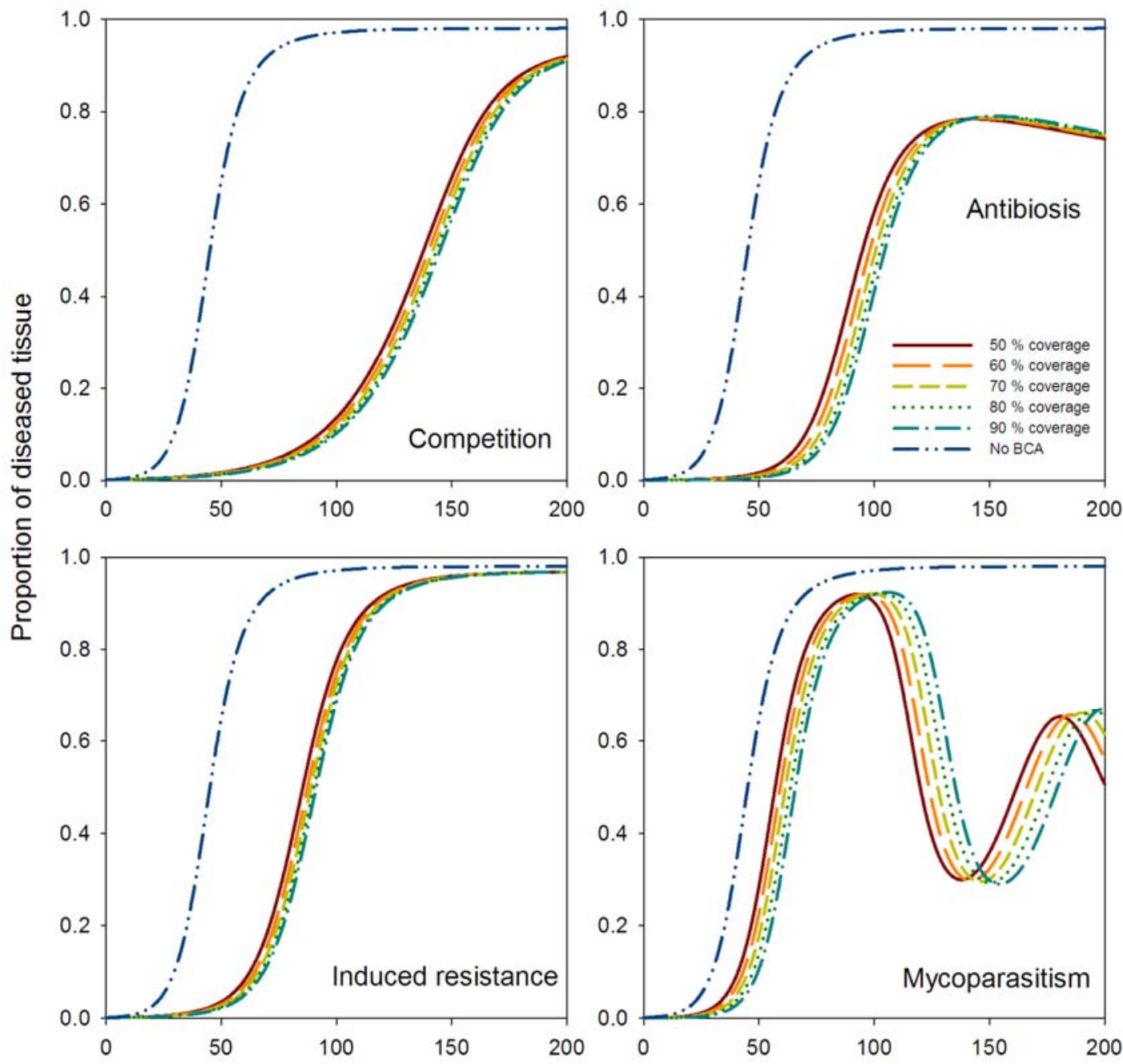

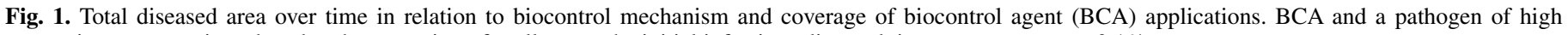
aggressiveness were introduced at the same time; for all cases, the initial infectious diseased tissue area was set to $0.1 \%$. 
When a BCA was introduced 28 days after the pathogen (total disease was then about $8 \%$ ), the effect of varying coverage on subsequent epidemic development was even more dependent on the biocontrol mechanism. For competition and induced resistance, varying coverage from 50 to $90 \%$ again had limited effect on epidemic development (Fig. 4). In contrast, increasing coverage from 50 to $90 \%$ for mechanisms involving either antibiosis or mycoparasitism had a marked effect in delaying epidemic development. For example, for a BCA involving competition, increasing the coverage from 50 to $90 \%$ led to an additional delay of only 7 days for total disease to reach $40 \%$ compared with the corresponding value of 24 days for a BCA involving antibiosis.

BCA application time. A delay in application of a BCA greatly reduced biocontrol efficacy for a BCA with a main mechanism of induced resistance or competition (Fig. 5). For instance, it took 97 and 64 days, respectively, for total disease to reach $40 \%$ when a BCA was applied 14 and 28 days after the pathogen, respectively, compared with 131 days when applied at the same time as the pathogen.

Efficacy of a BCA with more than one mechanism. Epidemic development of a high aggressive pathogen when a BCA with two mechanisms was applied at various delays after pathogen introduction is given in Figure 6. With the parameter values used, two combinations of biocontrol mechanisms were very effective in suppressing disease development: competition combined with either antibiosis or mycoparasitism. Irrespective of the application time (up to 28 days after pathogen introduction), a BCA with these combinations of mechanisms successfully prevented the pathogen from establishing in the host. Overall, a BCA combining induced resistance and antibiosis was least effective in controlling the disease (Fig. 6).

BCA application time did not noticeably affect biocontrol efficacy for any combination of mechanisms except for induced resistance and competition. Applying a BCA with induced resistance and competition 14 days after the pathogen introduction suppressed disease development greatly, but failed to do so when applied 28 days after the pathogen introduction (Fig. 6). Combining mycoparasitism with either antibiosis or induced resistance also suppressed disease development after an initial peak at about 110 to 140 days, with the amplitude of cycles seen with mycoparasitism much reduced by the second mechanism (Fig. 6). There was no advantage gained in combining the mechanisms of induced resistance and antibiosis irrespective of delay in time of application (Fig. 6). Reducing the strength of each component mechanism (i.e., by reducing the value of an appropriate parameter) may have a great impact on biocontrol efficacy. For example, the biocontrol efficacy achieved by a BCA combining competition and mycoparasitism varied greatly with $c_{1}$ and $c_{2}$ (Fig. 7).

\section{DISCUSSION}

A previous study derived qualitative characteristics of biocontrol systems described by an SIR model (equation 1) (13), focused
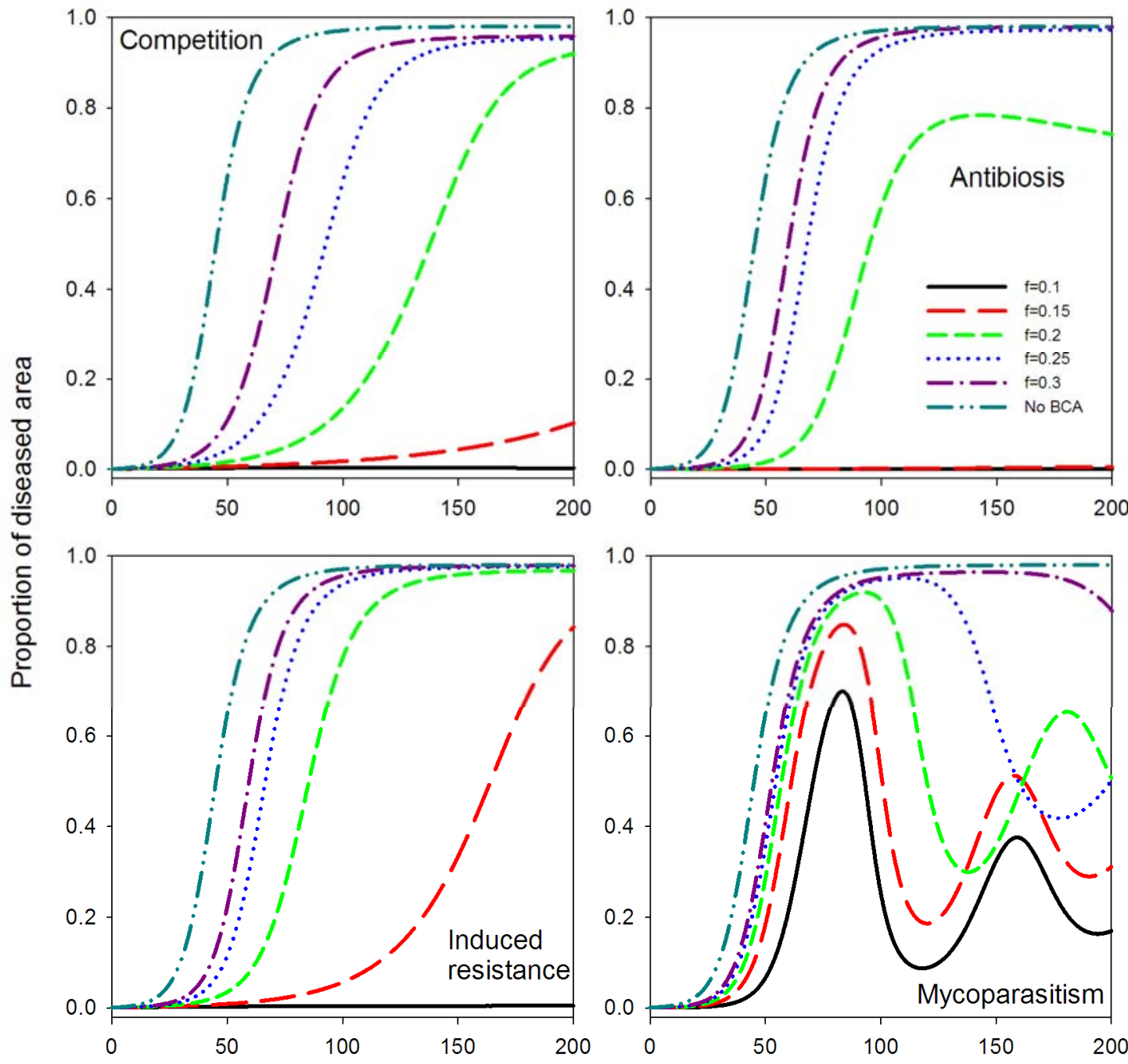

Fig. 3. Total diseased area in relation to the biocontrol agent (BCA) mortality rate. A BCA was applied at the same time as a pathogen of high aggressiveness at $50 \%$ coverage. 
on the basic reproductive number $\left(R_{0}\right)$ of disease, the system steady state and the parameter space that leads to steady states. It showed the importance of three biocontrol parameters in determining biocontrol outcomes - the rates of a BCA colonizing healthy $\left(c_{1}\right)$ and diseased $\left(c_{2}\right)$ host tissues and BCA mortality $(f)$. In the present study, we modified this model and conducted numerical evaluations to study the effects of BCA application time, coverage and combination of biocontrol mechanisms on epidemic development. Individual biocontrol mechanisms were defined by setting appropriate parameter(s) with numerical values (Table 1). The magnitudes of these values are debatable since there are few published quantitative data to estimate them, despite the huge volume of published research on biological control of foliar pathogens. However, we expect the main conclusions of our qualitative analysis to stand as long as the relative magnitudes of these values do not deviate significantly from those used in the present study.

As an individual mechanism, competition is most effective. This is probably due to the high colonization $c_{1}$ value used (0.4) in comparison to the mortality rate $(f=0.2)$ and the pathogen infection rate $(b=0.2)$. By definition, a BCA with competition as its main mechanism should have a greater ability of colonizing healthy tissue than a BCA with any other biocontrol mechanisms. However, $c_{1}$ reflects not only the ability of a BCA to colonize the immediate neighboring areas but probably, and more importantly, its ability to spread to new tissues. Only by the latter route can a
BCA fully realize the often-claimed advantage of a BCA being able to survive, reproduce, and colonize new host tissues. A recent study showed a limited spread of several BCAs among strawberry leaves (22). It should be noted that the requirement for a BCA to spread to and colonize new tissues is not necessary for some biocontrol systems, such as postharvest application of BCAs to control storage diseases. Under these circumstances, BCA survival $(f)$ is then critically important. For controlling foliar diseases in the field, the ability to spread to new tissues is essential if frequent inundative applications of BCAs are to be avoided. This characteristic of BCAs has often been neglected in biocontrol research and deserves more attention in future.

Despite the appealing nature of mycoparasitism, a BCA purely relying on this mechanism will not suppress disease development satisfactorily for commercial agriculture. This is because of the nature of disease development with a mycoparasitic BCA: disease development follows a damped oscillation before reaching a steady state. Thus, no matter how low the eventual disease (steady state value) may be, the disease has to increase initially to a much higher level. The amplitude and frequency of these oscillations depend on the model parameter values, and unless these are known precisely, predicting the timing of peaks is not possible. Unless the host can tolerate and recover sufficiently from severe disease, a BCA with mycoparasitism as its mechanism will have no practical use in commercial agriculture unless they are frequently applied, or combined with another mechanism as shown
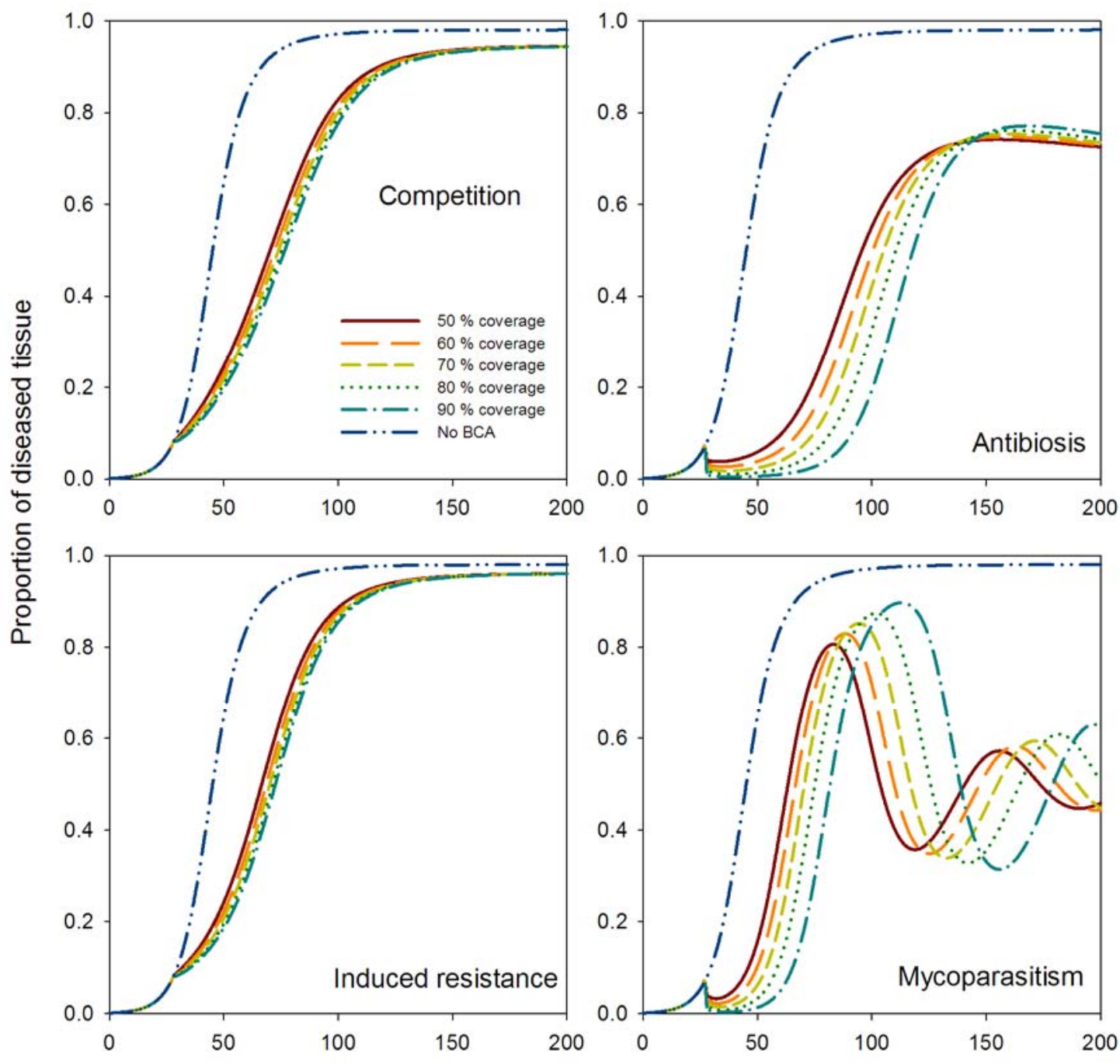

Fig. 4. Total diseased area in relation to biocontrol mechanism and coverage of biocontrol agent (BCA) applications when a BCA was applied 28 days after introduction of a high aggressiveness pathogen. 
later. The efficacy of a BCA with induced resistance or antibiosis as its single control mechanism is intermediate between BCAs with competition or mycoparasitism as their main mechanism.

The BCA mortality rate $(f)$ is critical in determining biocontrol efficacy. The mortality value of 0.2 used in the present study is within the range of observations of several BCAs $(2,7,9,24)$. For a BCA with induced resistance or antibiosis as the biocontrol mechanism, there is an added complication in determining another type of mortality (i.e., parameter $e$ ) - loss of induced resistance or antibiotic effects, relative to the rate of induced resistance or antibiosis $(d)$. If $e=0$ (without loss of resistance or antibiotics effect), biocontrol efficacy achieved with induced resistance or antibiosis would be much greater; in this scenario, disease suppression is greatest with induced resistance, assuming other parameter values remain the same as in Table 1 . In many cases, induced resistance may last between 4 to 14 days (12). In an extreme case, it may last longer than 100 days as observed on field-grown tomato plants treated with Trichoderma (19). Thus, for a BCA with induced resistance or antibiosis as its main mechanism, quantifying the relative magnitude of parameter $e$ is critical for predicting its biocontrol efficacy. This is particularly important given the current importance attached to induced resistance in biocontrol studies.

Contrary to intuition, increased BCA coverage from $50 \%$ to $90 \%$ does not lead to a great increase in biocontrol efficacy for most cases studied, even when a BCA was applied 28 days after pathogen introduction. This is probably due to a relatively higher BCA mortality rate $(f=0.2)$, in which the BCA colonized area reverts to healthy, susceptible tissue. Thus, a rapid turn-over in BCA-occupied tissues to healthy susceptible tissues renders the temporary sudden surge of BCA less effective. Thus, with small BCA mortality (i.e., equivalent to reducing and slow host growth), increasing coverage may be expected to become more important. For example, in extreme cases if there is no BCA mortality and host growth, a coverage value of $90 \%$ will mean that the maximum disease can only be $10 \%$ when the BCA was introduced before a pathogen. However, for high mortality and rapid host growth, increasing coverage is expected to be important only if the BCA is regularly applied to compensate mortality.

Application time of the BCA in relation to pathogen introduction is critically important only for BCAs with competition and, to a lesser extent, induced resistance as the main mechanism. For competition and induced resistance, a BCA does not affect diseased area directly but only reduces the susceptible area available to the pathogen. In contrast, when a BCA with antibiosis or mycoparasitism is applied, a proportion of the disease (at least $50 \%$ as assumed in the present study) is colonized by the BCA. Thus, the later it is applied, the greater will be converted (i.e., colonized or killed) by the BCA.

A BCA combining two main biocontrol mechanisms is much more effective in suppressing disease development than a BCA
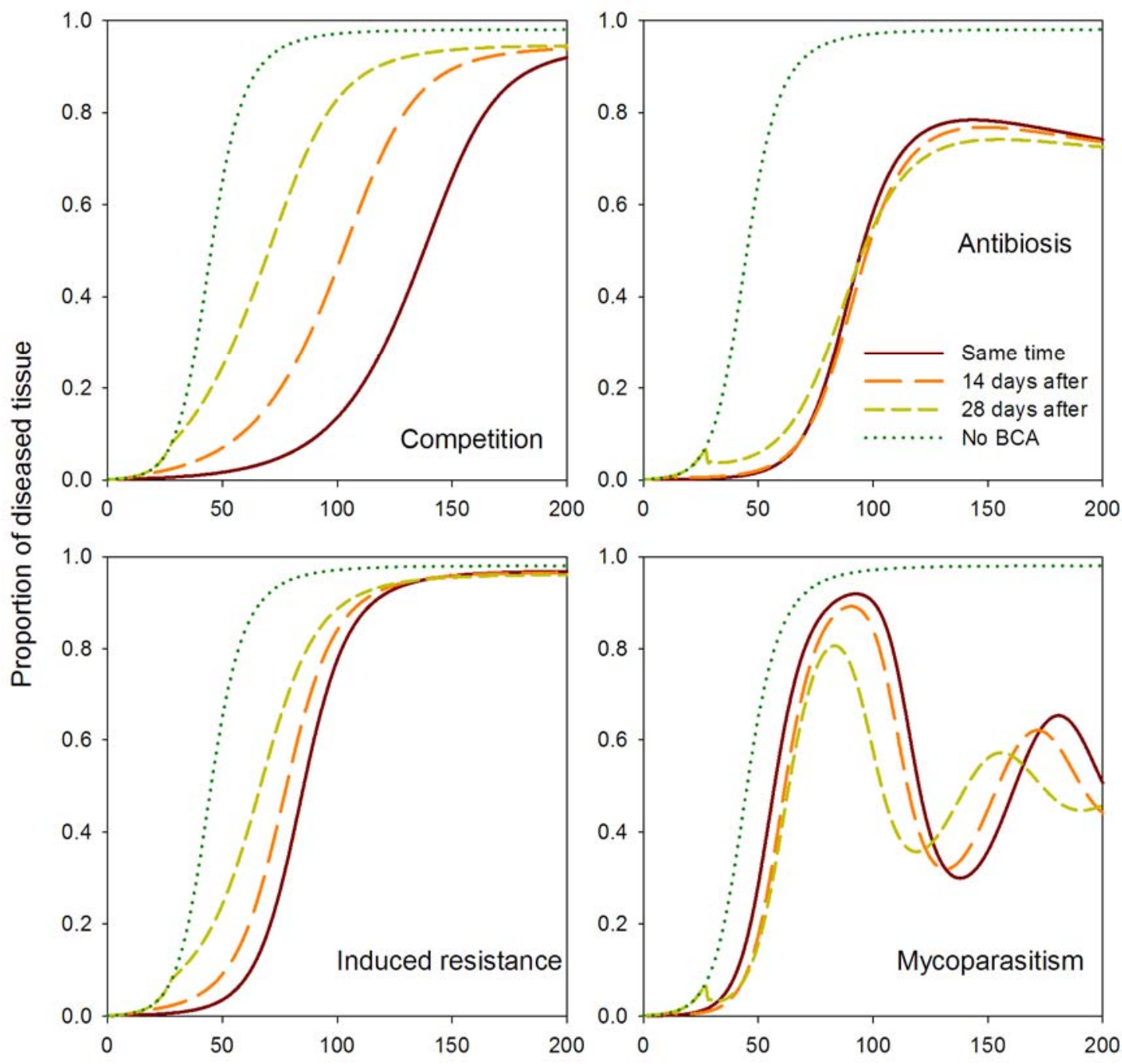

Fig. 5. Total diseased area in relation to biocontrol mechanism and the time lag when a biocontrol agent was applied at 50\% coverage after introduction of a pathogen of high aggressiveness with initial infectious diseased area of $0.1 \%$. 

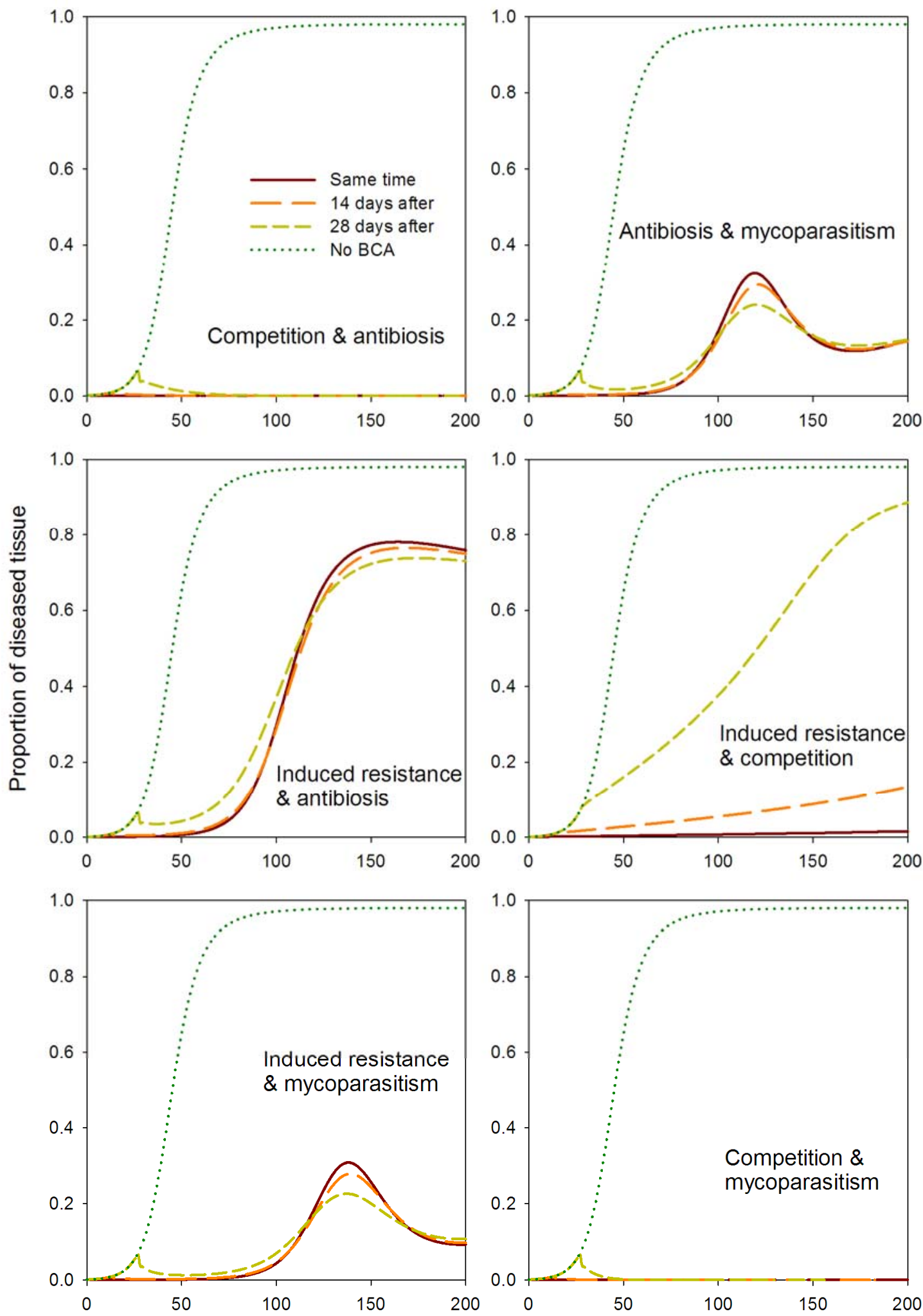

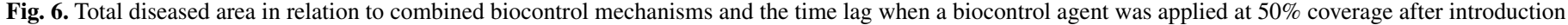
of a pathogen of high aggressiveness with initial infectious diseased area of $0.1 \%$.

with a single main mechanism. Combining higher rates for a $\mathrm{BCA}$ to colonies of both healthy $\left(c_{1}\right)$ and diseased $\left(c_{2}\right)$ tissues is the best strategy for suppressing disease development. Thus, a BCA combining competition with mycoparasitism (or antibiosis) led to completed suppression of a high aggressive pathogen whereas a BCA with only one of these mechanisms only managed to delay disease development. The damped oscillations observed in myco- parasitism are no longer observed. Furthermore, a BCA combining induced resistance with competition may control disease effectively but had to be applied early relative to the pathogen introduction for the reason discussed above. Combining induced resistance with antibiosis did not result in any improvement in disease suppression, which is probably due to the fact that both $c_{1}$ and $c_{2}$ are intermediate for this combination. However, it should 


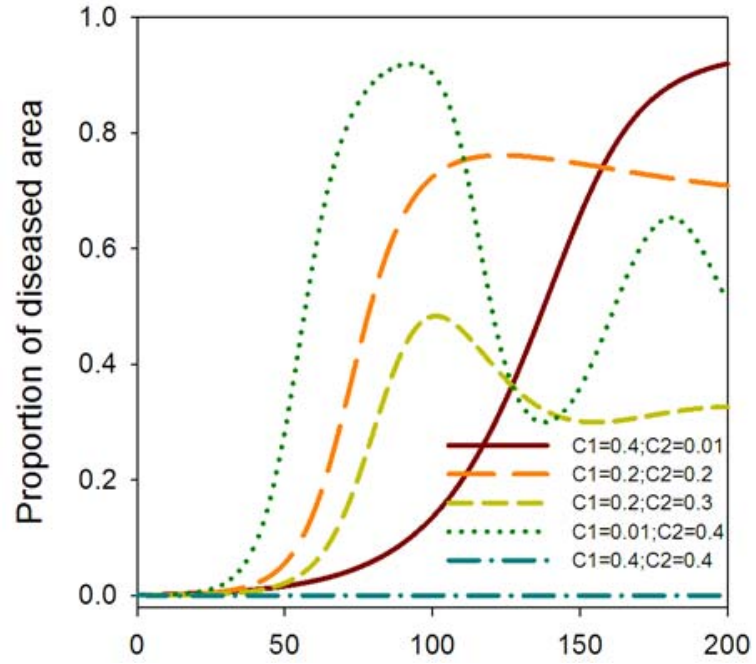

Fig. 7. Total diseased area when a biocontrol agent with both mycoparasitism and competition was applied (50\% coverage) at the same time as introduction of a pathogen of high aggressiveness.

be noted that the present conclusions are only applicable to a single BCA possessing two main biocontrol mechanisms, which is not the same as combining two BCAs, each with a single main mechanism. For the latter case, potential direct competition or interference between the two BCA has to be considered as well. We are currently extending the present model to investigate biocontrol with two BCAs.

This investigation suggests that a BCA with antibiosis or mycoparasitism as its main mechanism is unlikely to succeed unless it is applied frequently or combined with competition. As discussed before, disease control and hence the application strategy is conditioned on several key parameters: $c_{1}, c_{2}$, and $f$. Unfortunately there is little published direct information on these, especially on $c_{1}$. There are two aspects of $c_{1}$ that need to be considered: the colonization of immediate neighboring tissues in the same host unit and the colonization of (spreading to) new tissue units. Until there is reliable estimation on the relative magnitude of these parameter values for a given biocontrol system, it is unlikely that we could make reliable quantitative predictions of biocontrol efficacy and thus determine an efficient deployment strategy.

\section{ACKNOWLEDGMENTS}

This research was funded by the UK Pesticide Safety Directorate (PSD), Department of Environment Food and Rural Affairs (Defra) (Project number PS2119).

\section{LITERATURE CITED}

1. Arras, G., and Arru, S. 1997. Mechanism of action of some microbial antagonists against fungal pathogens. Ann. Microbiol. Enzimol. 47:97-120.

2. Boff, P. 2001. Epidemiology and Biological Control of Grey Mould in Annual Strawberry Crops. Wageningen University, Wageningen, The Netherlands.

3. Cabrefiga, J., and Montesinos, E. 2005. Analysis of aggressiveness of
Erwinia amylovora using disease-dose and time relationships. Phytopathology 95:1430-1437.

4. Elad, Y. 2000. Biological control of foliar pathogens by means of Trichoderma harzianum and potential modes of action. Crop Prot. 19:709-714.

5. Elad, Y., and Freeman, S. 2002. Biological control of fungal plant pathogens. Pages 93-109 in: The Mycota XI: Agricultural Applications, Springer-Verlag, Berlin.

6. Elad, Y., and Stewart, A. 2004. Microbial control of Botrytis spp. Pages 223-241 in: Botrytis: Biology, Pathology and Control. Y. Elad, ed. Kluwer Academic Publishers, Dordrecht, The Netherlands.

7. Elmer, P. A. G., and Köhl, J. 1998. The survival and saprophytic competitive ability of the Botrytis spp. antagonist Ulocladium atrum in lily canopies. Eur. J. Plant Pathol. 104:435-447.

8. Fravel, D. 2005. Commercialization and implementation of biocontrol. Annu. Rev. Phytopathol. 43:337-359.

9. Freeman, S., Minz, D., Kolesnik, I., Barbul, O., Zveibil, A., Maymon, M., Nitzani, Y., Kirshner, B., Rav-David, D., Bilu, A., Dag, A., Shafir, S., and Elad, Y. 2004. Trichoderma biocontrol of Colletotrichum acutatum and Botrytis cinerea and survival in strawberry. Eur. J. Plant Pathol. 110:361370.

10. Guetsky, R., Shtienberg, D., Elad, Y., and Dinoor, A. 2001. Combining biocontrol agents to reduce the variability of biological control. Phytopathology 91:621-627.

11. Guetsky, R., Shtienberg, D., Elad, Y., Fischer, E., and Dinoor, A. 2002. Improving biological control by combining biocontrol agents each with several mechanisms of disease suppression. Phytopathology 92:976-985.

12. Harman, G., Howell, C., Viterbo, A., Chet, I., and Lorito, M. 2004. Trichoderma species-Opportunistic, avirulent plant symbionts. Nat. Rev. Microbiol. 2:43-56.

13. Jeger, M. J., Jeffries, P., Elad, Y., and Xu, X. M. 2009. A generic theoretical model for biological control of foliar plant diseases. J. Theor. Biol. 256:201-214.

14. Kapat, A., Zimand, G., and Elad, Y. 1998. Effect of two isolates of Trichoderma harzianum on the activity of hydrolytic enzymes produced by Botrytis cinerea. Physiol. Mol. Plant Pathol. 52:127-137.

15. Kessel, G., Köhl, J., Powell, J., Rabbinge, R., and Van der Werf, W. 2005. Modelling spatial characteristics in the biological control of fungi at leaf scale: Competitive substrate colonization by Botrytis cinerea and the saprophytic antagonist Ulocladium atrum. Phytopathology 95:439448.

16. Knudson, G. R., and Hudler, G. W. 1987. Use of a computer simulation model to evaluate a plant disease biocontrol agent. Ecol. Model. 35:45-62.

17. Paulitz, T. C., and Belanger, R. R. 2001. Biological control in greenhouse systems. Annu. Rev. Phytopathol. 39:103-133.

18. Punja, Z. K., and Utkhede, R. S. 2003. Using fungi and yeasts to manage vegetable crop diseases. Trends Biotechnol. 21:400-407.

19. Seaman, A. 2003. Efficacy of OMRI-approved products for tomato foliar disease control. New York State Integrated Pest Management Program Publ. 129:164-167.

20. Taylor, D., Jarosz, A., Lenski, R., and Fulbright, D. 1998. The acquisition of hypovirulence in host-pathogen systems with three trophic levels. Am. Nat. 154:343-355.

21. Wilson, M. 1997. Biocontrol of aerial plant diseases in agriculture and horticulture: Current approaches and future prospects. J. Industrial Microbiol. Biotechnol. 19:188-191.

22. Xu, X.-M., Robinsona, J. D., Jeger, M., and Jeffries, P. 2010. Using combinations of biocontrol agents to control Botrytis cinerea on strawberry leaves under fluctuating temperatures. Biocontrol Sci. Technol. 20:359-373

23. Yedidia, I., Benhamou, N., Kapulnik, Y., and Chet, I. 2000. Induction and accumulation of PR proteins activity during early stages of root colonization by the mycoparasite Trichoderma harzianum strain T-203. Plant Physiol. Biochem. 38:863-873.

24. Yu, H., and Sutton, J. C. 1999. Density dynamics of Gliocladium roseum in relation to biological control of Botrytis cinerea in red raspberry. Can. J. Plant Pathol. 21:23-32. 